\title{
Inhibiting serotonin signaling through HTR2B in visceral adipose tissue improves obesity-related insulin resistance
}

\author{
Won Gun Choi, ${ }^{1}$ Wonsuk Choi,,${ }^{1,2}$ Tae Jung Oh, ${ }^{3}$ Hye-Na Cha, ${ }^{4}$ Inseon Hwang, ${ }^{1}$ Yun Kyung Lee, ${ }^{5}$ Seung Yeon Lee, ${ }^{1}$ Hyemi Shin, ${ }^{1}$ \\ Ajin Lim, ${ }^{1}$ Dongryeol Ryu, ${ }^{6}$ Jae Myoung Suh, ${ }^{1}$ So-Young Park, ${ }^{4}$ Sung Hee Choi, ${ }^{3,5}$ and Hail Kim \\ ${ }^{1}$ Graduate School of Medical Science and Engineering, Biomedical Research Center, KAIST, Daejeon, South Korea. ${ }^{2}$ Department of Internal Medicine, Chonnam National University Hwasun Hospital, Chonnam \\ National University Medical School, Hwasun, South Korea. ${ }^{3}$ Department of Internal Medicine, Seoul National University Bundang Hospital, Seoul National University College of Medicine, Seongnam, South \\ Korea. ${ }^{4}$ Department of Physiology, College of Medicine, Yeungnam University, Daegu, South Korea. ${ }^{5}$ Department of Internal Medicine, Seoul National University College of Medicine, Seoul, South Korea. \\ ${ }^{6}$ Department of Molecular Cell Biology, Sungkyunkwan University School of Medicine, Suwon, South Korea.
}

\begin{abstract}
Insulin resistance is a cornerstone of obesity-related complications such as type 2 diabetes, metabolic syndrome, and nonalcoholic fatty liver disease. A high rate of lipolysis is known to be associated with insulin resistance, and inhibiting adipose tissue lipolysis improves obesity-related insulin resistance. Here, we demonstrate that inhibition of serotonin (5-hydroxytryptamine [5-HT]) signaling through serotonin receptor 2B (HTR2B) in adipose tissues ameliorates insulin resistance by reducing lipolysis in visceral adipocytes. Chronic high-fat diet (HFD) feeding increased $H t r 2 b$ expression in epididymal white adipose tissue, resulting in increased HTR2B signaling in visceral white adipose tissue. Moreover, HTR2B expression in white adipose tissue was increased in obese humans and positively correlated with metabolic parameters. We further found that adipocyte-specific $\mathrm{Htr}$ b-knockout mice are resistant to HFD-induced insulin resistance, visceral adipose tissue inflammation, and hepatic steatosis. Enhanced 5-HT signaling through HTR2B directly activated lipolysis through phosphorylation of hormone-sensitive lipase in visceral adipocytes. Moreover, treatment with a selective HTR2B antagonist attenuated HFD-induced insulin resistance, visceral adipose tissue inflammation, and hepatic steatosis. Thus, adipose HTR2B signaling could be a potential therapeutic target for treatment of obesity-related insulin resistance.
\end{abstract}

\section{Introduction}

Obesity is a serious global health problem, affecting more than half a billion people worldwide (1). Importantly, obesity is a key underlying factor in several diseases related to insulin resistance, including type 2 diabetes, metabolic syndrome, and nonalcoholic fatty liver disease (NAFLD). Adipose tissue acts as an energy reservoir and endocrine organ to regulate systemic energy homeostasis. Obesity causes adipose tissue remodeling, characterized by adipocyte hyperplasia/hypertrophy, inflammation, and changes in adipose hormones, which leads to insulin resistance (2). Notably, expansion of visceral white adipose tissue (WAT) is closely associated with the development of insulin resistance, whereas expansion of subcutaneous WAT has no adverse effects on metabolic health $(3,4)$.

Serotonin (5-hydroxytryptamine [5-HT]) is a monoamine neurotransmitter that modulates many functions. 5-HT is synthesized from tryptophan by the sequential actions of tryptophan hydroxylase (TPH) and aromatic amino acid decarboxylase (AADC). Two isoforms of TPH are expressed in a mutually exclu-

Authorship note: WGC, WC, and TJO contributed equally to this work Conflict of interest: The authors have declared that no conflict of interest exists. Copyright: (5) 2021, American Society for Clinical Investigation.

Submitted: October 23, 2020; Accepted: October 5, 2021; Published: December 1, 2021. Reference information: / Clin Invest. 2021;131(23):e145331.

https://doi.org/10.1172/JCl145331. sive manner: TPH1 in peripheral nonneuronal tissues and TPH2 in neurons of central and enteric nervous systems $(5,6)$. Since 5-HT cannot cross the blood-brain barrier, central and peripheral 5-HT systems are functionally separated (7). 5-HT exerts its biological function through 5-HT receptors (HTRs). At least 14 HTRs have been identified in mammals, all of which are $G$ proteincoupled receptors (GPCRs) with the exception of HTR3, which is a ligand-gated ion channel (8).

5 -HT regulates multiple energy metabolism pathways in metabolic organs such as pancreatic $\beta$ cells, liver, skeletal muscle, and adipose tissues (9-16). In the obese state, 5-HT inhibits adaptive thermogenesis in brown and beige adipocytes $(12,13)$. Thus, genetic and chemical inhibition of peripheral 5-HT synthesis increases thermogenesis and protects against obesity-related metabolic dysfunction. In our previous study, we demonstrated a functional role for 5-HT in adipose tissues using adipocyte-specific Tph1 knockout (Tph1-FKO) mice $(13,17)$. The phenotypes of Tph1-FKO mice are largely attributable to the increased energy expenditure in brown adipose tissue (BAT) and inguinal white adipose tissue (iWAT). However, the function of 5-HT in epididymal white adipose tissue (eWAT) has not been directly tested in Tph1-FKO mice because of the profound effects of increased energy expenditure on eWAT. To overcome these limitations and explore the mechanism and function of 5-HT signaling in visceral adipocytes in detail, we examined receptor-specific roles of 5-HT in eWAT. 

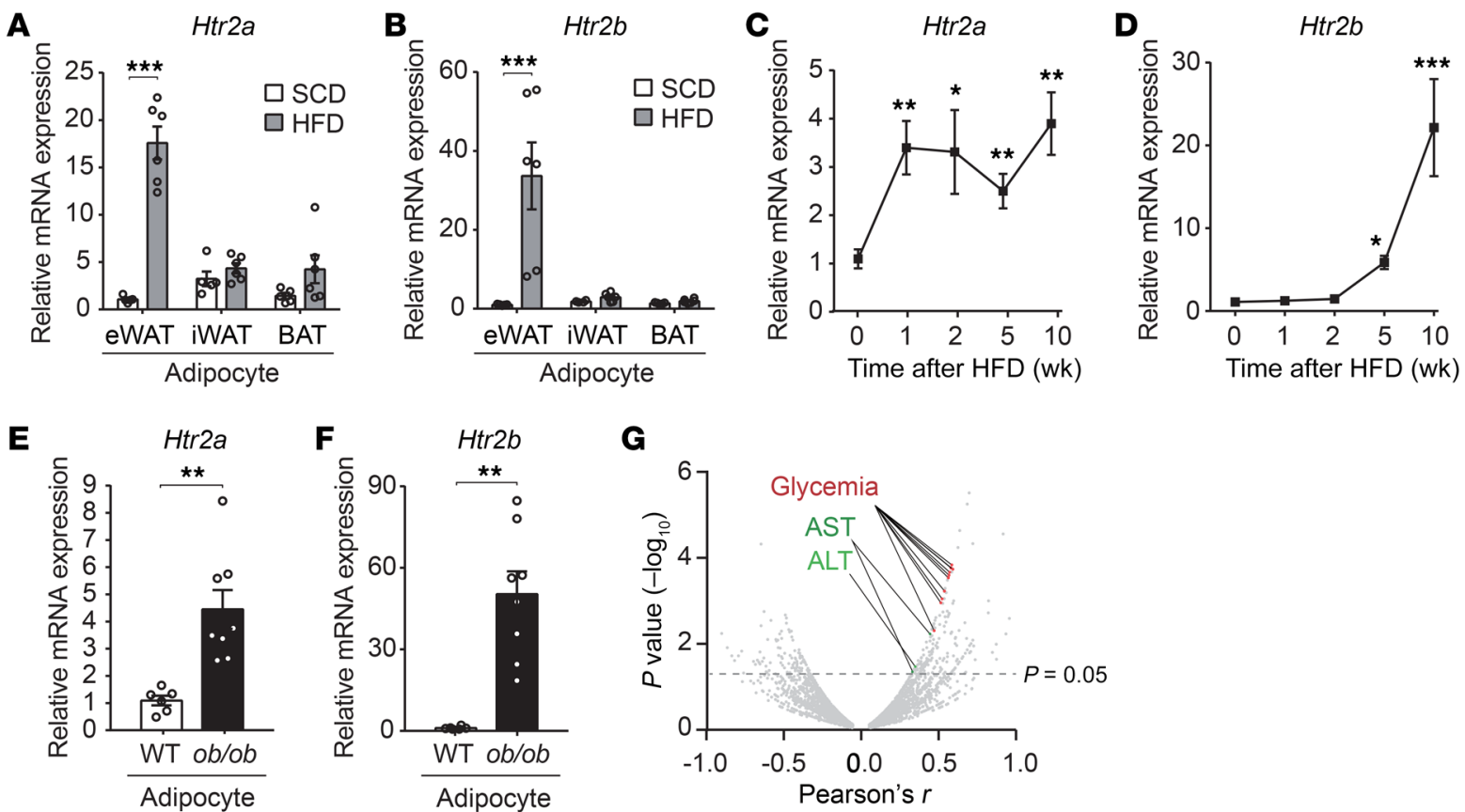

Figure 1. HTR2B signaling is upregulated in visceral adipose tissue of obese mice. (A and B) Twelve-week-old C57BL/6) mice were fed a SCD or HFD for 10 weeks. (A) Htr2a and (B) Htr2b mRNA expression in adipocytes isolated from eWAT, iWAT, and BAT, as assessed by qRT-PCR (SCD, $n=5 /$ group; HFD, $n=6$ /group). (C and D) (C) Htr2a and (D) Htr2b mRNA expression in eWAT from C57BL/6) mice after the indicated durations of HFD feeding, as assessed by qRT-PCR ( $n=7 /$ group). (E and F) Evaluation of 12-week-old $o b / o b$ mice. (E) Htr2a and (F) Htr2b mRNA expression in adipocytes isolated from eWAT, as assessed by qRT-PCR (WT, $n=6 /$ group; ob/ob, $n=8$ /group). (G) Correlation of phenotypes in the BXD mouse cohort with Htr2b expression in white adipose tissue. Glycemia (indicated by glucose tolerance tests) and blood levels of AST and ALT were significantly elevated in HFD-fed mice. Data are expressed as mean \pm SEM $\left({ }^{*} P<0.05 ;{ }^{* *} P<0.01 ;{ }^{* *} P<0.001\right.$, Student's $t$ test used in $\left.\mathbf{A}-\mathbf{F}\right)$.

Here, we demonstrate that 5-HT signaling through the HTR2B induces insulin resistance by increasing lipolysis in visceral adipocytes in an obese mouse model. Chronic high-fat diet (HFD) feeding increased $H \operatorname{tr} 2 b$ expression in eWAT. Thus, enhanced 5-HT signaling through HTR2B induced lipolysis by activating hormone-sensitive lipase (HSL). Inhibition of HTR2B signaling by adipocyte-specific $H t r 2 b$-KO or administration of a selective HTR2B antagonist improved systemic insulin sensitivity by reducing the release of free fatty acids (FFAs) from white adipocytes.

\section{Results}

HTR2B signaling is upregulated in visceral adipose tissue of obese mice. We first confirmed the expression of HTRs in visceral adipocytes (i.e., those isolated from eWAT) using publicly available RNA-seq data (18). Among HTR genes, Htr2a and Htr2b were abundantly expressed in visceral adipocytes and $H$ tr $2 b$ expression was increased upon HFD feeding for 9 weeks (Supplemental Figure 1A; supplemental material available online with this article; https://doi.org/10.1172/JCI145331DS1). Further quantitative reverse transcription-polymerase chain reaction (qRT-PCR) analyses using RNA isolated from different adipocytes revealed that HFD feeding for 10 weeks increased Htr $2 a$ and $H t r 2 b$ expression in visceral adipocytes but not in subcutaneous or brown adipocytes (i.e., those isolated from iWAT or BAT; Figure 1, A and B). Next, we assessed the dynamic expression patterns of Htr2a and $H$ tr $2 b$ in eWAT during HFD feeding. Htr $2 a$ expression increased beginning from 1 week after HFD feeding and remained increased throughout the HFD period (Figure 1C). In contrast, Htr $2 b$ expres- sion did not increase until 2 weeks after HFD feeding and started to increase after 5 weeks of HFD feeding (Figure 1D). Expression of both Htr2a and Htr2b was also upregulated in visceral adipocytes of $o b / o b$ mice (Figure 1, E and F). These results suggest a possible role of serotonergic signaling through HTR2A and HTR2B in eWAT under metabolic stress conditions. We previously reported the lipogenic actions of HTR2A in WAT $(13,17)$. However, a role for HTR2B in eWAT has not been elucidated, although $H \operatorname{tr} 2 b$ expression increased more robustly than Htr2a expression in visceral adipocytes of obese mouse models.

To gain insight into possible roles of HTR2B in WAT, we analyzed multi-omics data sets of the BXD mouse cohort, focusing on associations of Htr2b expression in WAT with clinical and molecular phenotypes (19). These analyses revealed that $H \operatorname{tr} 2 b$ mRNA expression levels were positively correlated with glycemia and blood levels of aspartate aminotransferase (AST) and alanine aminotransferase (ALT; Figure $1 \mathrm{G}$ and Supplemental Figure 1, B-D).

To further verify the physiological relevance of adipose HTR2B in humans, we assessed HTR2A and HTR2B expressions in human adipose tissues. Both HTR2A and HTR2B expressions were increased in visceral WAT of obese subjects, regardless of blood glucose levels (Figure 2, A and B). Interestingly, HTR2A and HTR $2 B$ expressions correlated with body mass index (BMI) in humans (Figure 2, C and D). Furthermore, consistent with our mouse data, both plasma AST and ALT concentrations correlated more highly with HTR $2 B$ expression than with HTR $2 A$ expression in human WAT (Figure 2, E-H). Taken together, these data suggest that HTR2B in WAT might have an important role under 
A

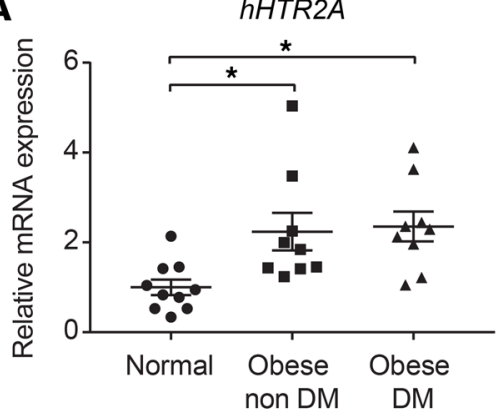

B

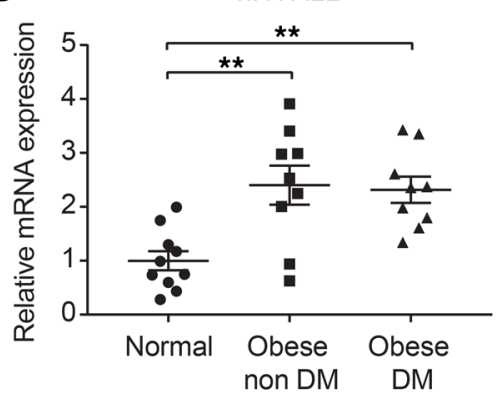

Figure 2. HTR2B signaling is upregulated in visceral adipose tissue of obese humans. (A-H) Potential role of HTR2A and HTR2B in human omental adipose tissue. (A and B) HTR2A and HTR2B expression in human omental fat from lean subjects $(n=10)$, nondiabetic obese subjects $(n=9)$, and diabetic obese subjects ( $n=$ 9). (C-H) Correlation of HTR2A and HTR2B expression in human omental fat with BMI (C and D), AST (E and F) and ALT (G and $\mathbf{H})(n=27)$. Pearson's $r$ correlation coefficient with corresponding $P$ values. Data are expressed as mean \pm SEM $\left({ }^{*} P<0.05\right.$; ${ }^{*} P<0.01$, 1-way ANOVA with post hoc Tukey's test used in $\mathbf{A}$ and $\mathbf{B}$ ).

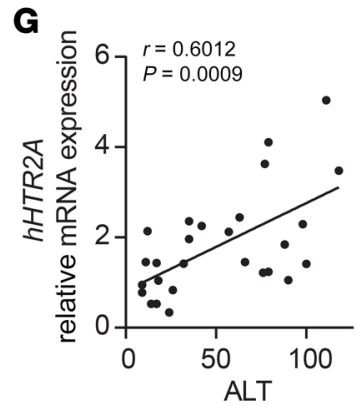

in $H$ tr $2 b$-FKO mice without a reduction in body weight or an increase in energy consumption.

To further evaluate the effect of Htr2b-FKO on insulin sensitivity, we performed hyperinsulinemic-euglycemic clamp. Glucose infusion rate and glucose turnover rate were increased in HFD-fed Htr2b-FKO mice (Figure 3, D and E). Moreover, insulin-stimulated AKT phosphorylation was enhanced in eWAT, liver, and muscle in Htr2b-FKO mice (Figure 3, F-H and Supplemental Figure 2, $\mathrm{M}-\mathrm{O}$ ). These results indicate that inhibiting adipose HTR2B signaling improves systemic insulin sensitivity in a HFD-induced mouse model of obesity.

Next, because obesity-induced insulin resistance is accompanied by adipose tissue remodeling, exemplified by adipocyte hypertrophy, macrophage infiltration, and proinflammatory cytokine production (2), we performed histological analyses of eWAT from HFD-fed Htr2b-FKO

chronic obesity conditions, prompting us to explore the metabolic function of serotonergic signaling through HTR2B in visceral WAT in greater detail.

Inhibiting adipose HTR2B signaling ameliorates HFD-induced systemic insulin resistance. To investigate the functional role of 5-HT signaling through HTR2B in WAT in vivo, we generated adipocyte-specific Htr2b-KO (Adiponectin-Cre ${ }^{+/} \mathrm{Htr}_{2} \mathrm{~b}^{\text {f/fl}}[\mathrm{Htr} 2 \mathrm{~b}-$ FKO]) mice and induced obesity by feeding them a HFD for 10 weeks (Supplemental Figure 2A). Glucose tolerance and body weight of Htr2b-FKO mice were comparable to those of littermate control $\left(H t r 2 b^{f / f l}\right)$ mice when fed a standard chow diet (SCD) (Supplemental Figure 2, B and C). Upon HFD feeding, Htr2b-FKO mice showed improved glucose tolerance despite a similar gain in body weight (Figure 3A and Supplemental Figure 2B). Furthermore, blood glucose concentrations of $H$ tr $2 b$-FKO mice were lower than control mice during insulin tolerance tests, and plasma insulin concentrations were also decreased compared with control mice (Figure 3, B and C). However, body composition, food intake, and energy expenditure in HFD-fed Htr $2 b$-FKO mice were comparable to those in control mice on HFD (Supplemental Figure 2, D-K). In addition, oral lipid tolerance test revealed that lipid absorption was not affected in the gut of HFD-fed Htr2b-FKO mice (Supplemental Figure 2L). These data suggest that, unlike the case in Tph1-FKO mice, HFD-induced insulin resistance is ameliorated mice. Adipocyte size was more uniform and the proportion of hypertrophic adipocytes was reduced in eWAT of HFD-fed Htr2bFKO mice, but average adipocyte size was comparable (Figure 4, A and B, and Supplemental Figure 3A). Macrophage infiltration was diminished and $F 4 / 80$ expression was decreased in eWAT of HFD-fed Htr2b-FKO mice (Figure 4, C and D). However, no histological change was detected in iWAT or BAT of Htr2b-FKO mice (Supplemental Figure 3, B and C). qRT-PCR exhibited the expressions of genes involved in proinflammatory pathways was reduced and adiponectin expression was increased in visceral adipocytes and stromal vascular fraction (SVF) isolated from eWAT of HFDfed Htr2b-FKO mice (Figure 4, E-H). The expressions of genes involved in adipogenesis and lipolysis were not changed in eWAT of HFD-fed Htr $2 b$-FKO mice (Supplemental Figure 3, D-H). These results indicate that inhibiting adipose HTR2B signaling resolves key characteristics of insulin resistance in EWAT in a mouse model of diet-induced obesity.

5-HT signaling through HTR2B promotes lipolysis in eWAT. To further explore the metabolic pathways underlying the attenuated systemic insulin resistance in HFD-fed Htr2b-FKO mice, we performed global metabolomics profiling on the plasma of HFD-fed Htr2b-FKO mice and control mice. Among 827 metabolites detected in 9 super-pathways (Supplemental Figure 4A), 151 metabolites were significantly changed in Htr2b-FKO mice, with 

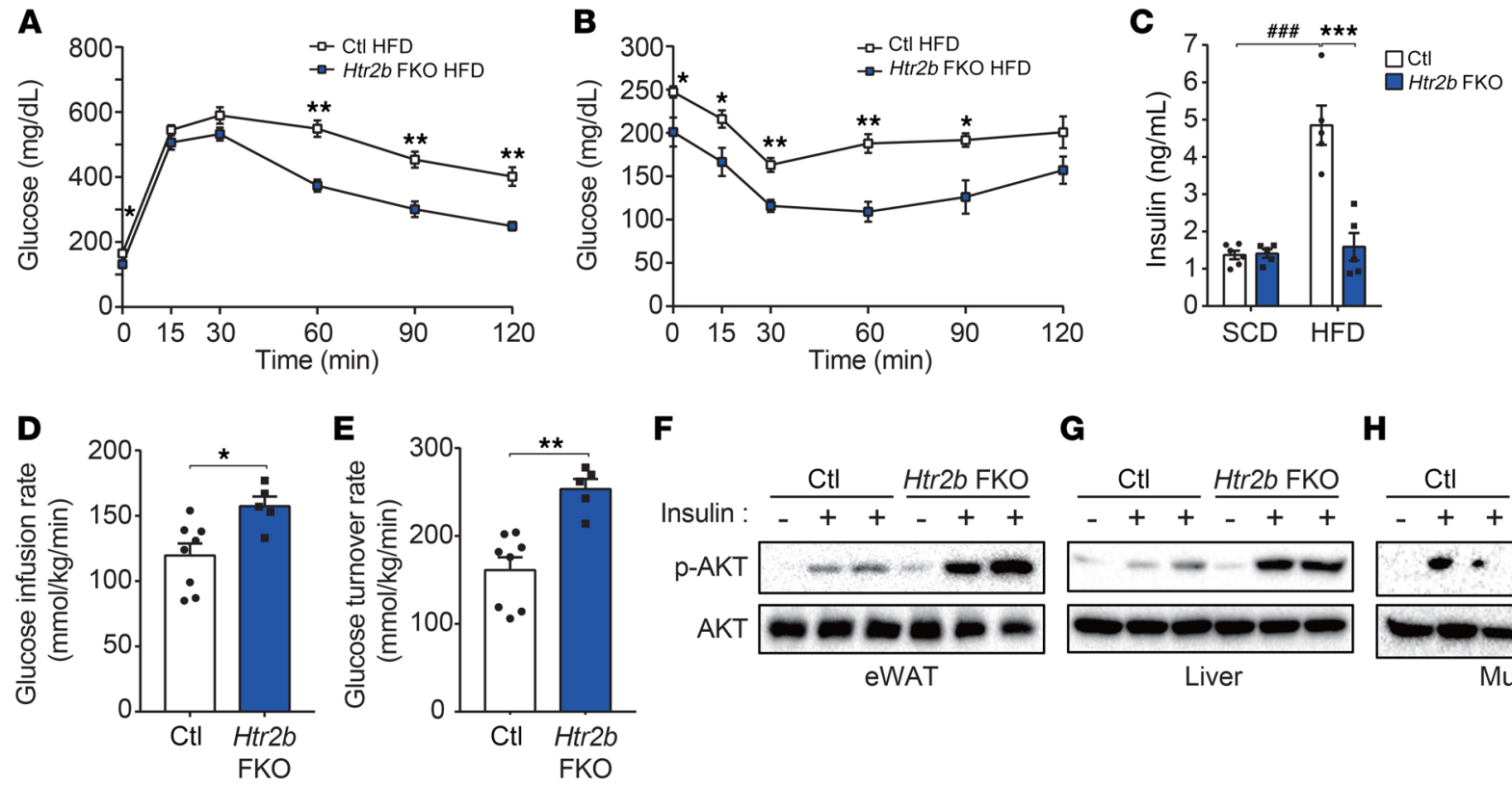

$\mathbf{F}$

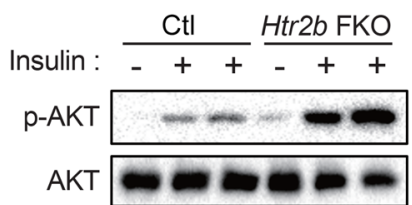

eWAT
G

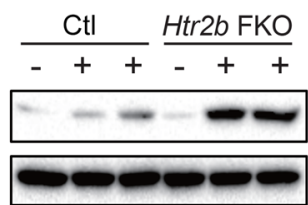

Liver
1

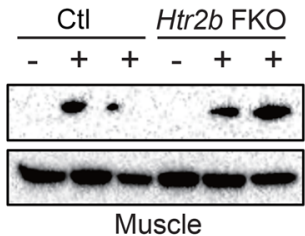

Figure 3. Htr2b-FKO mice are protected from HFD-induced systemic insulin resistance. (A-H) Twelve-week-old control and $H$ tr2b-FKO mice were fed a SCD or HFD for 10 weeks. (A) IPGTT after 16 hours fasting ( $n=8 /$ group) and (B) IPITT after 6 hours fasting ( $n=5 / g r o u p)$ in HFD-fed mice. (C) Plasma insulin levels (SCD-fed control mice, $n=6$; SCD-fed Htr2b-FKO mice, $n=5$; HFD-fed mice, $n=5 /$ group). (D and E) Glucose infusion rate (D) and glucose turnover rate (E) in HFD-fed mice, measured by hyperinsulinemic-euglycemic clamp (control, $n=8 /$ group; Htr2b-FKO, $n=5 /$ group). (F-H) Insulin-induced AKT phosphorylation in eWAT (F), liver (G), and skeletal muscle (H) of HFD-fed control and Htr2b-FKO mice, as assessed by Western blotting. Data are expressed as

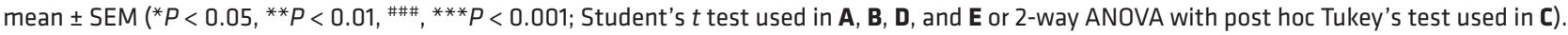

57 metabolites exhibiting an increase and 94 metabolites exhibiting a decrease (Supplemental Figure 4, B-D). An analysis of the 151 significantly changed metabolites, performed by enrichment on 62 subpathways containing 5 or more metabolites out of the 827 detected metabolites, identified the top 10 enriched subpathways (Figure 5A). Interestingly, 3 subpathways related to longchain fatty acids (FAs) were included in these top 10 enriched subpathways, and most long-chain FAs were decreased in Htr $2 b$-FKO mice compared with control mice (Figure 5A and Supplemental Figure 4, E-G). These data suggest a decrease in plasma FFA levels in $H t r 2 b$-FKO mice. Indeed, plasma FFA concentration was decreased in the plasma of HFD-fed Htr $2 b$-FKO mice (Figure 5B).

HFD feeding is known to increase plasma FFA concentrations, which are primarily regulated by adipocyte lipolysis in WAT and are positively correlated with insulin resistance (20). Therefore, we examined whether 5-HT signaling through HTR2B can directly regulate lipolysis in visceral adipocytes. Primary visceral adipocytes isolated from eWAT of HFD-fed mice were treated with 5-HT or the HTR2B agonist BW 723C86. Both 5-HT and BW $723 \mathrm{C} 86$ activated HSL by inducing HSL phosphorylation (Figure 5, C and D and Supplemental Figure 4, H and I). 5-HT-induced HSL phosphorylation was blunted by the HTR2B antagonist SB 204741 (Figure 5E and Supplemental Figure 4J). Moreover, BW $723 \mathrm{C} 86$ increased glycerol release from primary visceral adipocytes (Figure 5F). However, 5-HT did not induce phosphorylation of HSL in primary subcutaneous adipocytes isolated from iWAT of HFD-fed mice (Supplemental Figure 4K). In addition, neither 5-HT nor BW 723C86 induced HSL phosphorylation in primary visceral adipocytes isolated from SCD-fed mice (Supplemental Figure $4 \mathrm{~L}$ ). These data suggest that increased $H \operatorname{tr} 2 b$ expression is necessary for the induction of HSL phosphorylation in visceral adipocytes by 5-HT. Consistently, i.p. injection of 5-HT increased HSL phosphorylation in eWAT of HFD-fed control mice but not in eWAT of HFD-fed Htr2b-FKO mice (Figure 5G and Supplemental Figure 4M). These results suggest that adipose 5-HT signaling through HTR2B promotes lipolysis in visceral adipocytes of obese mice through HSL phosphorylation and decreases systemic insulin sensitivity by increasing plasma FFA levels.

5-HT phosphorylates HSL through the Ca2+-cGMP-PKG cascade in visceral adipocytes. Because HTR2B is a $\mathrm{G}_{\mathrm{q}}$ protein-coupled receptor ( $\left.G_{q} P C R\right)$, when activated, it increases intracellular $\mathrm{Ca}^{2+}$, which can lead to the production cAMP and cGMP and thereby activate protein kinase A (PKA) and protein kinase G (PKG), respectively (21-23). To identify the downstream mechanism by which 5-HT induces HSL phosphorylation, we treated visceral adipocytes isolated from HFD-fed mice with the intracellular $\mathrm{Ca}^{2+}$ chelator BAPTA-AM. BAPTA-AM reduced 5-HT-induced HSL phosphorylation in visceral adipocytes, indicating that HSL phosphorylation in response to 5-HT stimulation depends on intracellular $\mathrm{Ca}^{2+}$ levels (Figure $5 \mathrm{H}$ and Supplemental Figure $4 \mathrm{~N}$ ).

HSL phosphorylation is mediated by PKA and PKG $(24,25)$. Since PKA is a major regulator of HSL phosphorylation in adipocytes, we first examined whether 5-HT-induced HSL phosphorylation depends on PKA. Arguing against this, neither the PKA inhibitor KT 5720 nor the adenylyl cyclase (AC) inhibitors SQ 22536 and KH7 inhibited 5-HT-induced HSL phosphorylation in visceral adipocytes (Supplemental Figure 4, O and P). Instead, the selective PKG inhibitor KT 5823 and selective guanylyl cyclase (GC) inhibitors ODQ and NS 2028 reduced 5-HT-induced HSL phosphorylation in visceral adipocytes, indicating that 5-HT- 

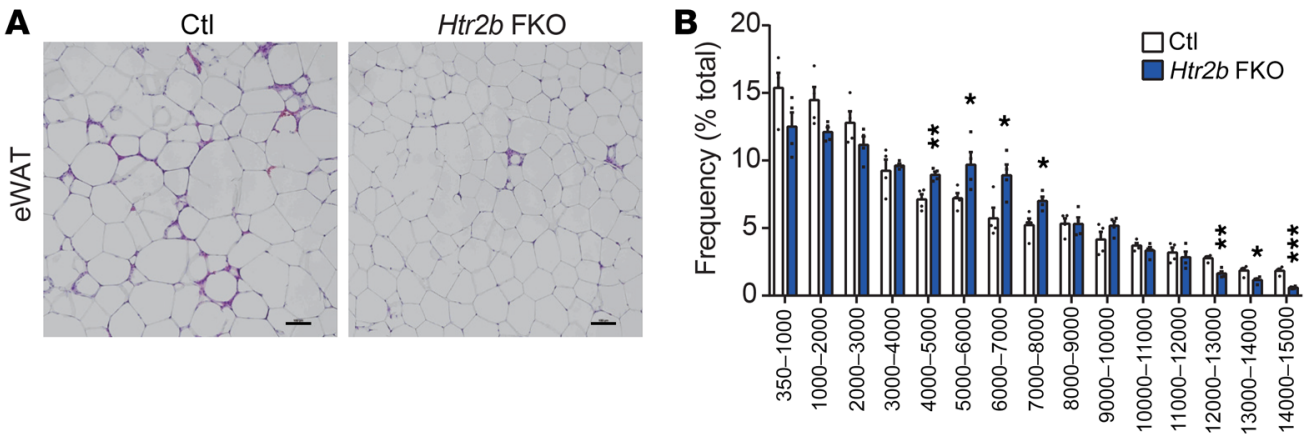

Adipocyte area $\left(\mu \mathrm{m}^{2}\right)$
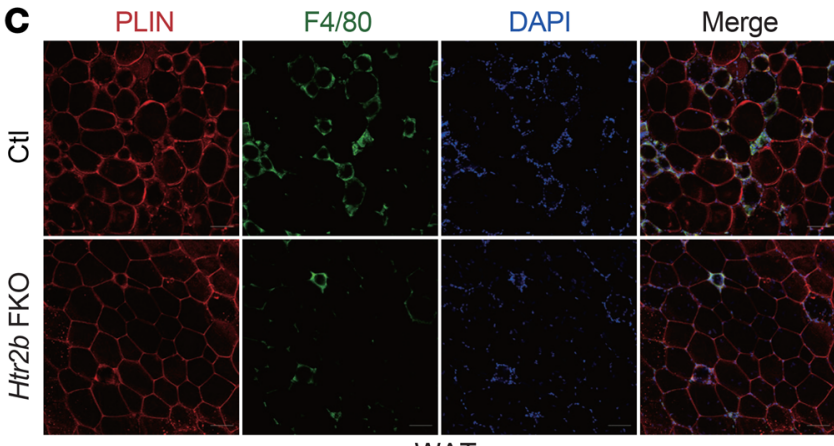

eWAT
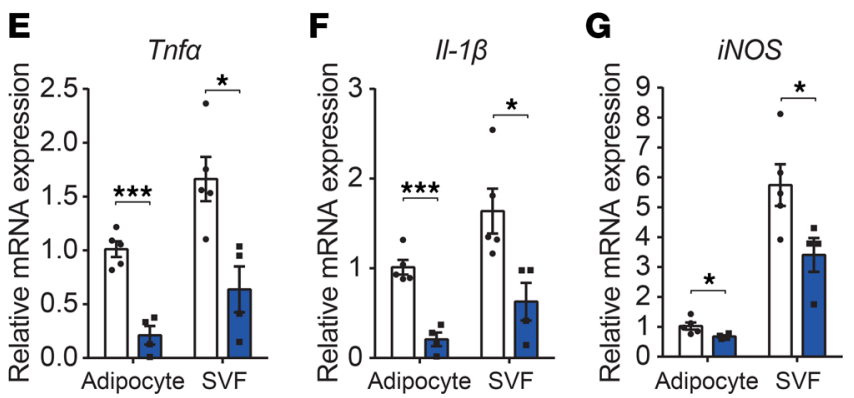

D

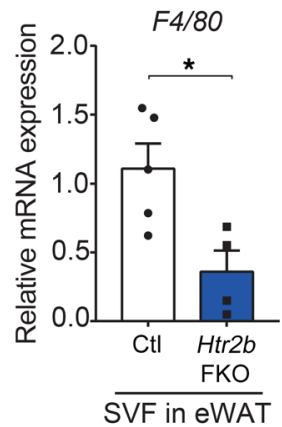

H

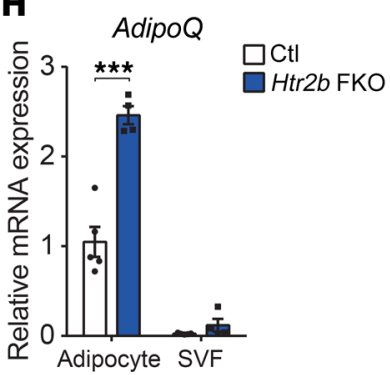

Figure 4. HFD-induced adipose tissue insulin resistance is ameliorated in Htr2b-FKO mice. (A) Representative eWAT histology, assessed by H\&E staining. Scale bars: 100 $\mu \mathrm{m}$. (B) Histogram showing the distribution of adipocyte frequency according to diameter in eWAT ( $n=$ 4/group, 5 images per mouse). (C) Immunofluorescence (IF) staining of the eWAT marker perilipin 1 (PLIN1, red) and macrophage marker $\mathrm{F} 4 / 80$ (green), and staining of nuclei with DAPI (blue). (D) F4/80 mRNA expression in SVF isolated from eWAT, as assessed by qRT-PCR (control, $n=5$ /group; Htr2b-FKO, $n=$ 4/group). (E-H) Relative expression of mRNA for proinflammatory pathway-related genes $(\mathbf{E}-\mathbf{G})$ or adiponectin (H), as assessed by qRT-PCR in adipocytes and SVF isolated from eWAT (control, $n=$ 5/group; Htr2b-FKO, $n=4$ /group). Data are expressed as mean \pm SEM $\left({ }^{*} P<0.05,{ }^{* *} P<0.01,{ }^{* * *} P<0.001\right.$, Student's $t$ test used in $\mathbf{B}, \mathbf{D}-\mathbf{H})$. induced HSL phosphorylation is mediated by cGMP-PKG rather than cAMP-PKA (Figure 3, I and J and Supplemental Figure 4, Q and R). Taken together, our data indicate that adipose 5-HT signaling through HTR2B activates a $\mathrm{Ca}^{2+}-\mathrm{cGMP}-\mathrm{PKG}$ cascade that increases HSL phosphorylation and ultimately increases lipolysis in visceral adipocytes of obese mice (Figure $5 \mathrm{~K}$ ).

Inhibiting adipose HTR2B signaling ameliorates HFD-induced hepatic steatosis. During insulin resistance, increased lipolysis in adipocytes increases hepatic FFA influx and thereby promotes hepatic steatosis (26). In quantitative terms, $60 \%$ of triglycerides (TGs) in the livers of patients with NAFLD originates from WAT (27). Because adipose 5-HT signaling through HTR2B promotes lipolysis in visceral adipocytes and thereby increases plasma FFA concentrations, we evaluated the hepatic phenotype of HFD-fed $H t r 2 b$-FKO mice and found that lipid accumulation and hepatic TG levels were decreased in Htr $2 b$-FKO mice compared with control mice (Figure 6, A and B).

To monitor changes in hepatic metabolism secondary to $H$ tr $2 b$-FKO, we performed gene expression analyses on an extensive array of metabolic markers. Expression of genes involved in FA uptake and TG synthesis were generally decreased in the livers of $H$ tr $2 b$-FKO mice compared with that in control mice (Figure 6,
C and D). However, with the exception of Srebp1c, genes involved in de novo lipogenesis were not significantly changed in the livers of HFD-fed Htr2b-FKO mice (Supplemental Figure 5A). Plasma AST and ALT levels were reduced in HFD-fed Htr $2 b$-FKO mice, a finding that aligns with the association between adipose $H$ tr $2 b$ expression and plasma AST and ALT levels in the BXD mouse cohort and in humans (Figure 1G, Figure 2, F and H, and Figure $6 \mathrm{E})$. These results suggest that the protective effect of $H t r 2 b$-FKO mice against HFD-induced hepatic steatosis is attributable to the reduction in FA flux from WAT to liver.

Pharmacological inhibition of HTR2B improves HFD-induced metabolic dysfunction. Taken together, data from Htr2b-FKO mice establish that inhibiting adipose HTR2B signaling protects against HFD-induced metabolic dysfunctions, including systemic insulin resistance, visceral adipose tissue inflammation, and hepatic steatosis. These results led us to evaluate the possibility of selective HTR2B antagonism as a novel therapeutic strategy for obesityrelated metabolic dysfunctions. To this end, we fed mice HFD for 10 weeks and then administered SB 204741 daily via i.p. injection while continuing HFD feeding for 3 weeks (Supplemental Figure 6A). Treatment of mice with SB 204741 improved glucose tolerance and reduced plasma insulin levels without affecting body 
A

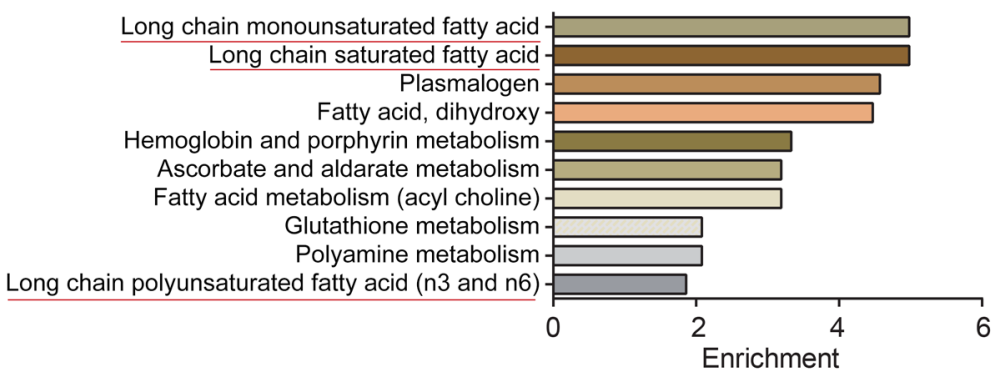

B

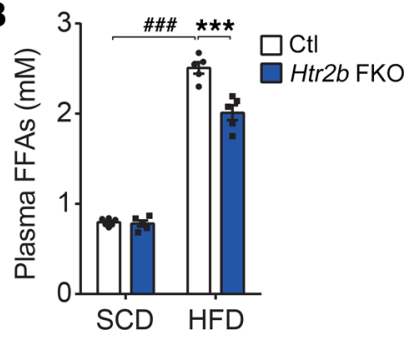

C

5-HT

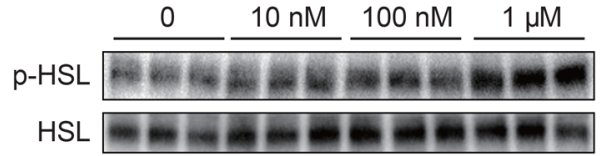

E

5-HT $-\quad-\quad-\quad-+++++++$

SB $204741-\quad-\quad+++\quad-\quad-+++$

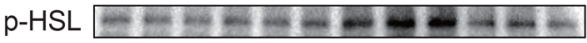

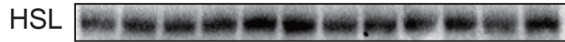

$\mathbf{H}$

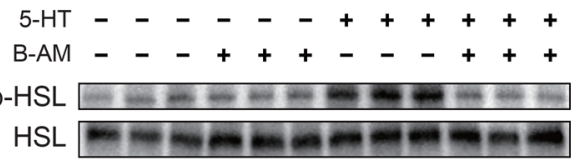

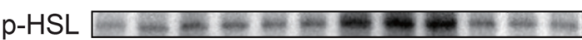

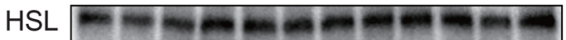

D

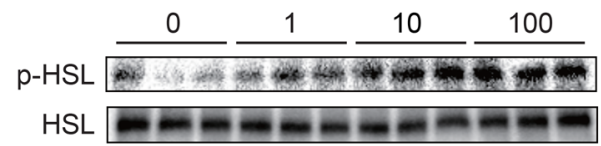

$\mathbf{F}$

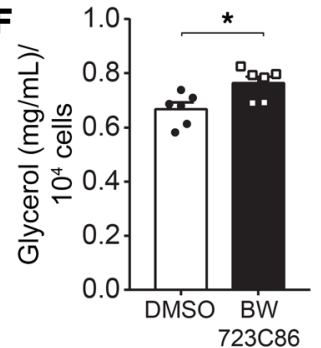

I
G

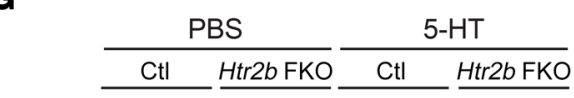

p-HSL $=-m-m-\infty-\infty-\infty-m$

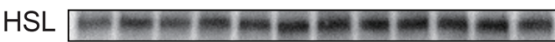

$\mathbf{J}$

5-HT - - - - - - - - +++++++++

$\mathrm{ODQ}---+++------+++--$

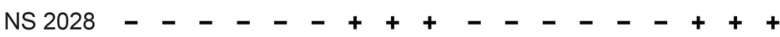

p-HSL $\square=0$

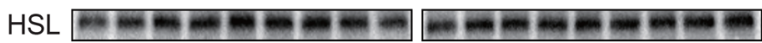

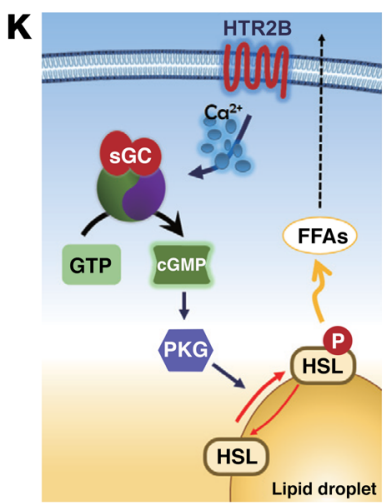

Figure 5. 5-HT signaling via HTR2B promotes lipolysis in visceral adipocytes of obese mice. (A and B) Twelve-week-old control and $H t r 2 b$-FKO mice were fed HFD for 10 weeks. (A) Metabolomics was performed on plasma for significant enrichment. Only the top 10 enrichment subpathways are shown ( $n=6 /$ group). (B) Plasma FFA levels in HFD-fed mice (control and Htr2b-FKO, $n=5$ ) and SCD-fed mice (control, $n=6 ; H t r 2 b$-FKO, $n=5$ ). (C-E and G-J) Western blot of pHSL and HSL using adipocytes isolated from eWAT of HFD-fed mice. (C-D) Treatment with different concentrations of 5-HT (C) or BW 723C86 (D) for 15 minutes. (E) Treatment with $1 \mu \mathrm{M}$ 5-HT for 15 minutes, with or without pretreatment for 30 minutes with $10 \mu \mathrm{M}$ SB 204741. (F) Assay of glycerol release in conditioned media after treatment with $10 \mu \mathrm{M} \mathrm{BW} 723$ C86 for 6 hours using adipocytes isolated from eWAT of HFD-fed mice. (C) Treatment with $4 \mu \mathrm{g} / \mathrm{kg}$ 5-HT (i.p.) for 15 minutes. (H) Treatment with $1 \mu \mathrm{M}$ 5-HT for 15 minutes, with pretreatment with $1 \mu \mathrm{M}$ BAPTA-AM for 30 minutes. (I) Treatment with $1 \mu \mathrm{M}$ 5-HT for 15 minutes, with pretreatment with $1 \mu \mathrm{M} \mathrm{KT} 5823$ for 30 minutes. (J) Treatment with $1 \mu \mathrm{M}$ 5-HT for 15 minutes, with pretreatment with $10 \mu \mathrm{M}$ ODQ and $10 \mu \mathrm{M}$ NS 2028 for 30 minutes. (K) Graphical summary. Data are expressed as mean \pm SEM $\left({ }^{*} P<0.05\right.$, \#\#\#, ${ }^{* * *} P<0.001$; Student's $t$ test used in $\mathbf{F}$ or 2-way ANOVA with post hoc Tukey's test used in B).

weight or body composition (Figure 7, A and B and Supplemental Figure 6, B-E). The effects of SB 204741 treatment closely resembled those of Htr2b-FKO mice: it decreased the proportion of hypertrophic adipocytes without change of average adipocyte size; decreased visceral adipose tissue inflammation; reduced plasma FFA levels; improved hepatic steatosis accompanied by downregulation of expression of genes involved in FA uptake, TG synthesis, and de novo lipogenesis; decreased plasma AST and ALT levels; and was characterized by the absence of histological changes in iWAT and BAT (Figure 7, C-M and Supplemental Figure 6, F-K).
We further validated the efficacy of SB 204741 on Htr $2 b$-FKO mice with the same strategy (Supplemental Figure 7A). Unlike WT mice, SB 204741 treatment did not affect glucose tolerance, plasma insulin level, and plasma FFA levels in Htr2b-FKO mice (Supplemental Figure 7, B-D). In addition, SB 204741 treatment did not induce any histological changes in eWAT, liver, iWAT, and BAT of Htr $2 b$-FKO mice (Supplemental Figure 7, E-H). These results demonstrate that HTR2B antagonism improves metabolic dysfunction in HFD-fed mice, likely through mechanisms in common with those of $H$ tr $2 b$-FKO mice. 
A

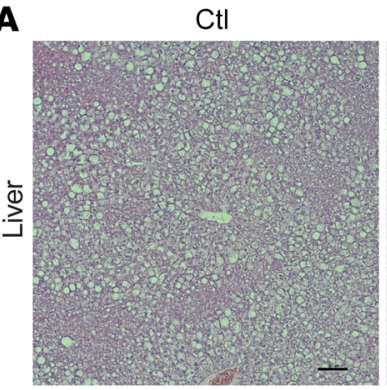

Htr2b FKO

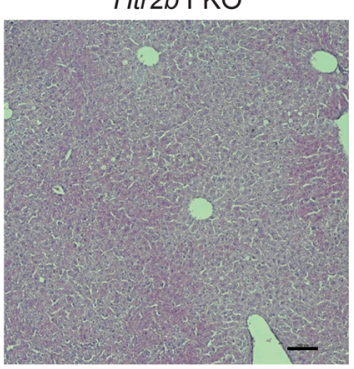

B

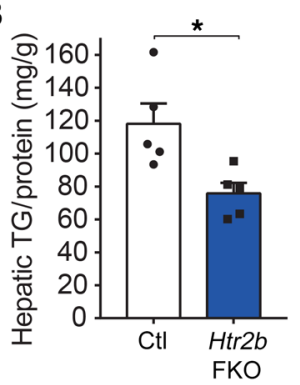

C
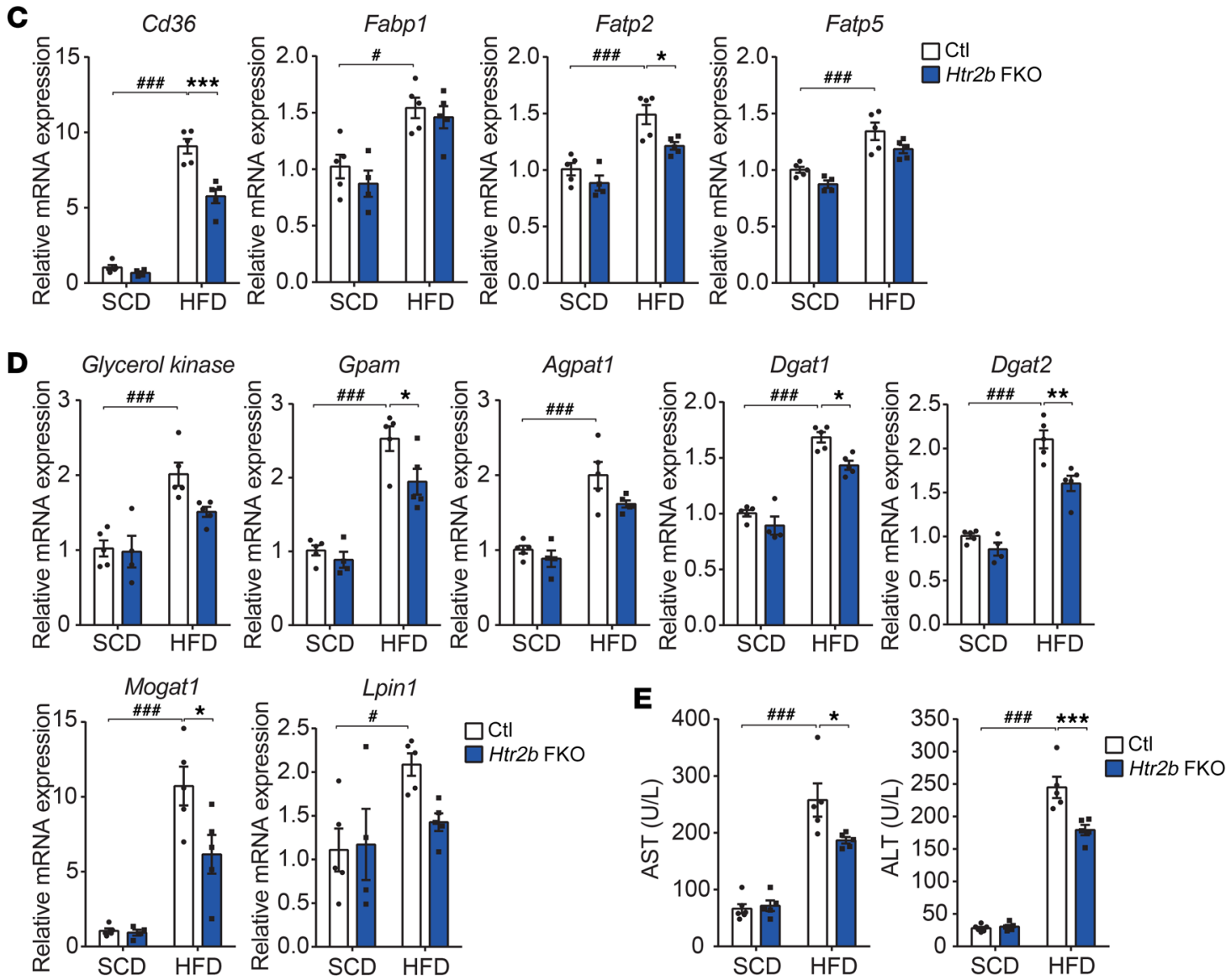

Figure 6. HFD-induced hepatic steatosis is ameliorated in Htr2b-FKO mice. (A-E) Twelve-week-old control and Htr2b-FKO mice were fed a SCD or HFD for 10 weeks. (A) Representative liver histology of HFD-fed control and Htr2b-FKO mice, as assessed by H\&E staining. Scale bars: $100 \mu$ m. (B) Hepatic TC levels ( $n=5$ /group). (C and D) Relative expression of mRNA for genes involved in FA uptake (C) and TC synthesis (D) in the liver, as assessed by qRT-PCR ( $n=5$ /group). (E) Plasma AST and ALT levels (SCD-fed control mice, $n=6$; SCD-fed Htr2b-FKO mice, $n=5$; HFD-fed mice, $n=5 /$ group). Data are expressed

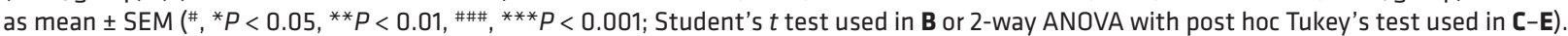

\section{Discussion}

Peripheral 5-HT is emerging as a regulator of energy metabolism during obesity-related insulin resistance, exerting its roles in liver and adipose tissues $(11-13,17,28)$. Adipocyte-derived 5-HT inhibits adaptive thermogenesis in brown and subcutaneous adipose tissues, whereas gut-derived 5-HT regulates hepatic lipid accumulation through the gut-liver axis. However, a direct functional role of 5-HT in visceral WAT has not yet been elucidated. Here, we demonstrate that inhibition of adipose HTR2B signaling ameliorates systemic insulin resistance, visceral adipose tissue inflammation, and hepatic steatosis accompanied by decreased plasma
FFA levels. Mechanistically, adipose 5-HT signaling via HTR2B promotes lipolysis by inducing HSL phosphorylation through the $\mathrm{Ca}^{2+}-$ cGMP-PKG axis in visceral adipocytes. On the basis of these results, we propose that adipose HTR2B signaling acts as a regulator of systemic insulin resistance by promoting lipolysis.

A high plasma 5-HT concentration is positively correlated with BMI and glycated hemoglobin in human subjects (29). Similarly, the concentration of plasma 5-HIAA, the final metabolite of 5-HT, is positively correlated with fasting glucose levels and is higher in subjects with metabolic syndrome than in those without it (30). In the present study, we verified that WAT HTR2B expression is increased 

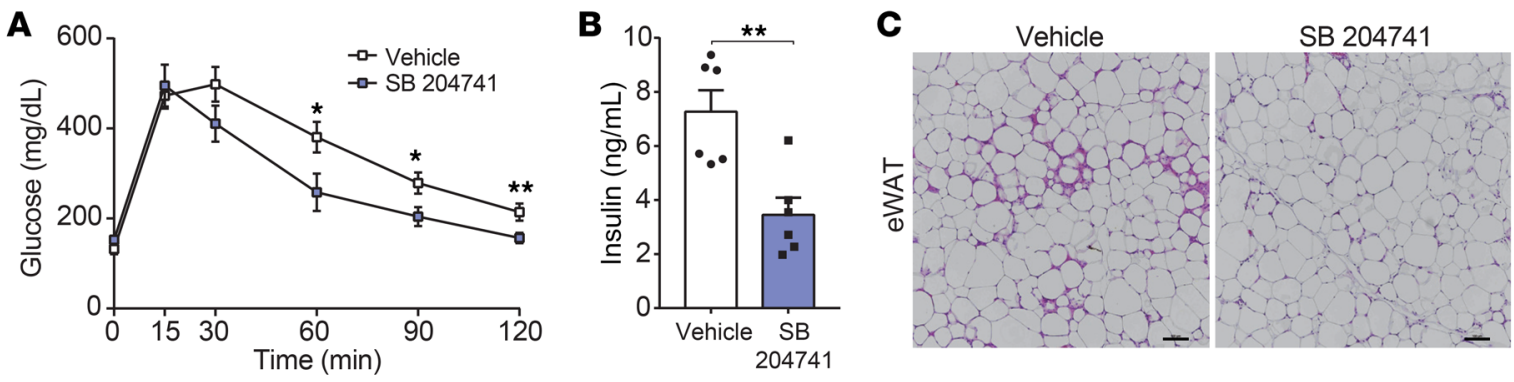

D

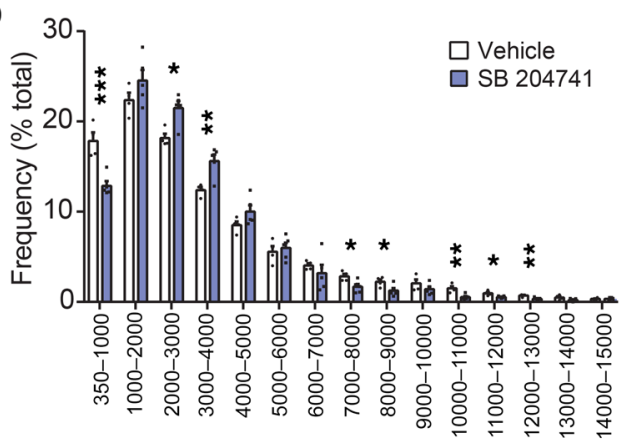

E PLIN

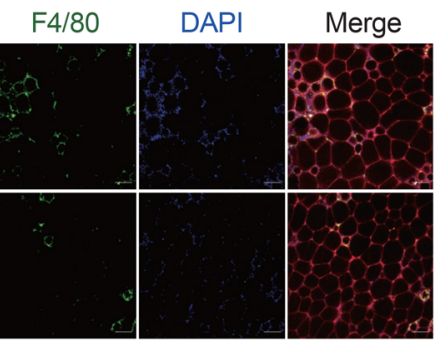

eWAT

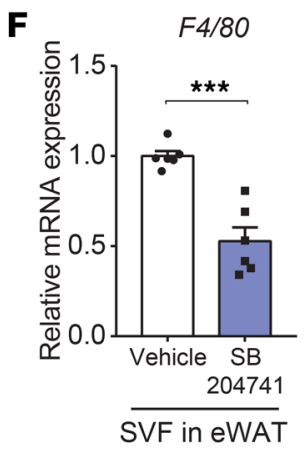

Adipocyte area $\left(\mu \mathrm{m}^{2}\right)$
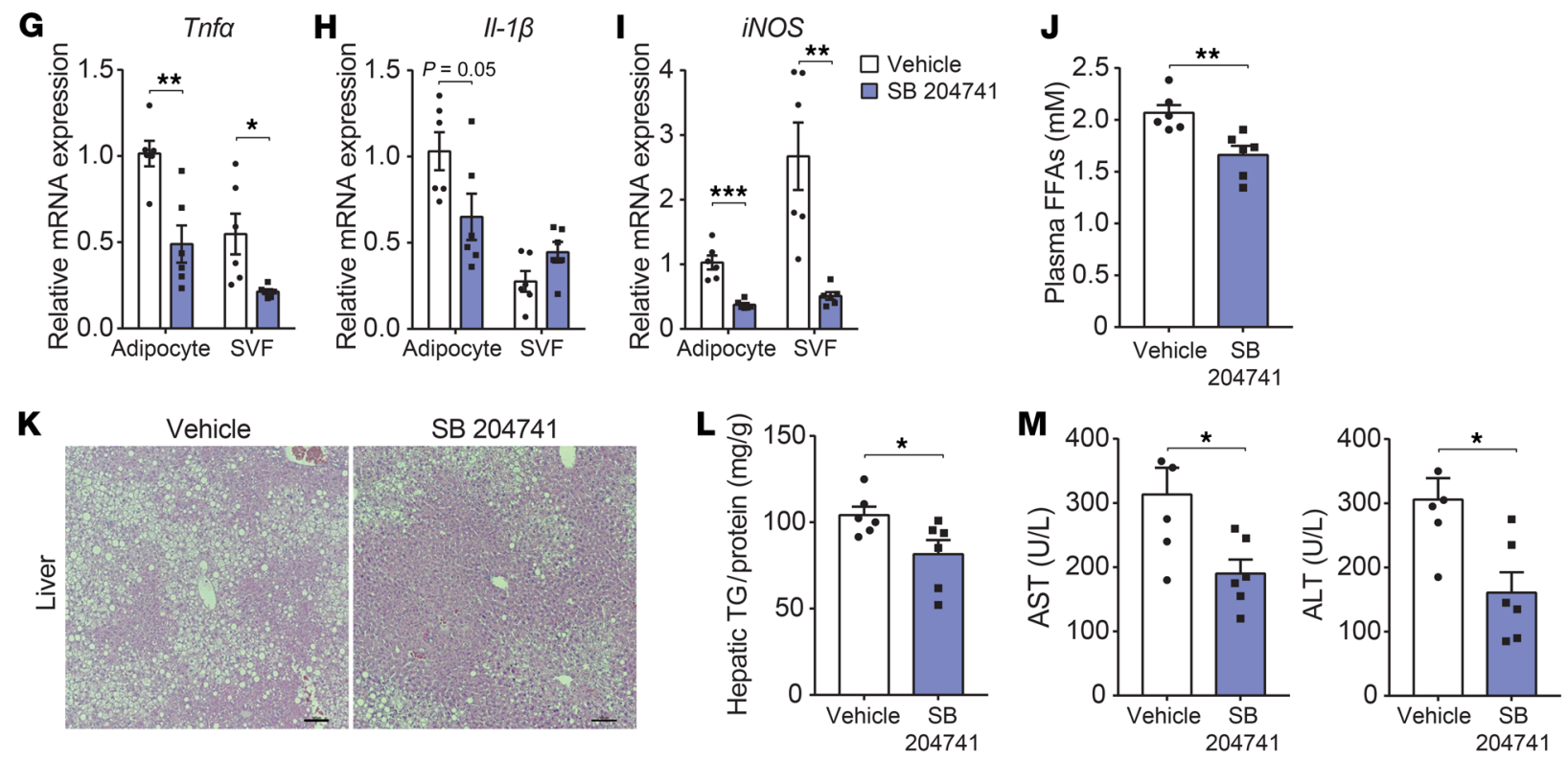

Figure 7. Pharmacological inhibition of HTR2B improves HFD-induced metabolic dysfunction. (A-M) Twelve-week-old mice were fed a HFD for 10 weeks and treated with vehicle or SB 204741 daily via i.p. injection for 3 weeks while continuing HFD feeding. (A) IPGTTs of HFD-fed vehicle and SB 204741-treated mice ( $n=6$ /group). (B) Plasma insulin levels in HFD-fed mice treated with vehicle or SB 204741 ( $n=6 /$ group). (C) Representative eWAT histology in HFD-fed mice treated with SB 204741, as assessed by H\&E staining. Scale bars: $100 \mu \mathrm{m}$. (D) Histogram showing the distribution of adipocyte frequency according to diameter (vehicle, $n=4$; SB 204741, $n=5$, 5 images per mouse) (E) IF staining for perilipin 1 (red) and F4/80 (green) in eWAT from HFDfed mice treated with vehicle or SB 204741, and counterstaining of nuclei with DAPI (blue). (F) F4/80 mRNA expression in SVF isolated from eWAT, as assessed by qRT-PCR (vehicle, $n=6$; SB 204741, $n=6$ ). (G-I) Relative expression of mRNA for genes involved in proinflammatory pathways in adipocytes and SVF isolated from eWAT, as assessed by qRT-PCR ( $n=6$ /group). (J) Plasma FFA levels ( $n=6 /$ group). (K) Representative liver histology in HFD-fed mice treated with vehicle or SB 204741, as assessed by H\&E staining. Scale bars: $100 \mu \mathrm{m}$. (L) Hepatic TC levels ( $n=6 / g r o u p)$. (M) Plasma AST and ALT levels ( $n=$ 6/group). Data are expressed as mean \pm SEM $\left({ }^{*} P<0.05,{ }^{* *} P<0.01,{ }^{* * *} P<0.001\right.$, Student's $t$ test used in $\mathbf{A}, \mathbf{B}, \mathbf{D}, \mathbf{F}-\mathbf{J}, \mathbf{L}$, and $\left.\mathbf{M}\right)$.

in mouse models of obesity (HFD-fed mice, $o b / o b$ mice) and humans (Figure 1, B and F and Figure 2B). Furthermore, HTR2B expression is positively correlated with glycemia and plasma AST and ALT levels in a BXD mouse cohort (Supplemental Figure 1, B-D) and with BMI and plasma AST and ALT levels in humans (Figure 2, D, F, and
$\mathrm{H})$. These data are generally supportive of the idea that adipose 5-HT signaling through HTR2B contributes to the pathogenesis of obesity-related metabolic dysfunction in both mice and humans.

A high lipolysis rate is associated with low insulin sensitivity, and inhibiting adipose tissue lipolysis, whether by knocking out 
adipocyte-specific adipose triglyceride lipase (ATGL) or through HSL haploinsufficiency, improves obesity-related insulin resistance without causing a change in body weight $(31,32)$. However, there are limitations to using ATGL and HSL as therapeutic targets for obesity-related insulin resistance, since these enzymes are physiologically essential for maintaining energy homeostasis. In this study, we demonstrated that adipose HTR2B signaling activates lipolysis specifically in visceral adipocytes under obese conditions (Figure 5, C, D, and F and Supplemental Figure 4, H, I, $\mathrm{K}$, and L). Furthermore, Htr2b-FKO mice improved insulin resistance only in the HFD-fed state, without causing metabolic changes in the SCD-fed state (Figure 3A and Supplemental Figure 2C). Our findings highlight the potential of adipose HTR2B signaling as a novel therapeutic target for obesity-related insulin resistance through modulating effects on lipolysis in visceral adipose tissue.

NAFLD, which refers to a group of conditions characterized by excessive fat accumulation in the liver, progresses from hepatic steatosis to steatohepatitis, cirrhosis, and hepatocellular carcinoma (33). Despite the increasing global burden of NAFLD, no pharmacological agents have been approved for its treatment. A previous study reported that HTR2B signaling in hepatic stellate cells promotes fibrosis by inducing the expression of transforming growth factor $\beta 1$ (34). In the present study, we showed that hepatic steatosis and elevated plasma AST and ALT levels induced by HFD feeding were ameliorated in $H t r 2 b$-FKO mice (Figure 6, A, B, and E). In addition, administering a selective HTR2B antagonist after 10 weeks of HFD feeding produced similar outcomes (Figure 7, K-M). Considering that liver-specific $H t r 2 b-\mathrm{KO}$ mice exert no protective effect against HFD-induced hepatic steatosis (11), these effects are likely mainly attributable to HTR2B actions in adipose tissue. Taken together, our results and those of others suggest that systemic HTR2B antagonism, which simultaneously inhibits hepatic steatosis and fibrosis, may be an effective therapeutic strategy against NAFLD. Drugs that modulate 5-HT signaling pathways are widely used to treat various human diseases (35-40) and thus could be repurposed to treat NAFLD-related steatohepatitis and fibrosis, underscoring the importance of evaluating the therapeutic efficacy of systemic HTR2B antagonism in this setting.

In conclusion, our findings verify a role for adipose HTR2B signaling in energy homeostasis and suggest that inhibition of adipose HTR2B signaling may be a promising strategy for the targeted treatment of obesity-related insulin resistance. Future studies evaluating the significance of this pathway in humans with obesity and insulin resistance are needed.

\section{Methods}

All materials and reagents are listed in Supplemental Table 1.

Animal experiments. Htr $2 b^{\mathrm{t} / \mathrm{ll}}$ mice were crossed with AdiponectinCre mice to generate adipose tissue-specific Htr2b-knockout (Htr2bFKO) mice. Htr $2 b$ floxed mice and Adiponectin-Cre mice have been previously described $(10,41)$. C57BL/6J mice were purchased from Charles River Japan. Mice were housed in a specific pathogen-free barrier facility under a 12-hour light/dark cycle. Chow and water were provided ad libitum. At 12 weeks of age, mice were fed a SCD or a HFD (60\% of calories from fat). Food consumption was measured by weighing the food in individual cages every other day. Mice were euthanized by cervical dis- location. Fluid and lean and fat mass were measured in live mice using a body composition analyzer (LF50 BCA Analyzer; Bruker).

Adipocyte isolation and stromal vascular fractionation. Visceral adipose tissue (epididymal adipose tissue) was isolated from 22-week-old lean or obese mice. Adipocytes and the stromal vascular fraction (SVF) of epididymal adipose tissue were separated by collagenase digestion by mincing and then incubating tissue in isolation buffer (3\% BSA in HBSS) containing $1 \mathrm{mg} / \mathrm{mL}$ collagenase type II (LS004176; Worthington) in a $37^{\circ} \mathrm{C}$ shaking incubator for 30 minutes. The digested tissue was filtered through a $100-\mathrm{mm}$ mesh filter (BD Bioscience). Mature adipocytes and connective tissues were separated from the cell pellet by centrifuging at $800 \mathrm{~g}$ for 10 minutes at $4^{\circ} \mathrm{C}$ and then washing cells twice with culture media. Pelleted stromal vascular cells and adipocytes in the upper supernatant were resuspended in TRIzol reagent.

Primary adipocyte culture. Isolated primary adipocytes from adipose tissue were cultured in DMEM supplemented with $100 \mu \mathrm{g} / \mathrm{mL}$ penicillin/streptomycin at $37^{\circ} \mathrm{C}$ in a humidified $5 \% \mathrm{CO}_{2}$ atmosphere. Chemical treatments were applied to floating adipocytes on a shaker at 120 to $150 \mathrm{rpm}$. After treatment, the infranatant solution was removed using a $1 \mathrm{~mL}$ pipette, and primary adipocytes were prepared for isolation of RNA or protein.

Blood profiling. After fasting for 6 hours, mice were sacrificed and blood samples were obtained by cardiac puncture. Blood profiling was performed by GC Pharma (Green Cross Corporation). ALT and AST were detected using a modified International Federation of Clinical Chemistry UV method (without pyridoxal phosphate or sample blank). FFA was detected with an in vitro enzymatic colorimetric assay (NEFA HR.II; Wako) using a Cobas 8000 clinical analyzer with a c702 module (Roche).

Metabolic analysis. For measurement of metabolic rates, mice housed individually in an 8-chamber, open-circuit Oxymax/CLAMS (Columbus Instruments Comprehensive Lab Animal Monitoring System) apparatus were assessed for 72 hours in the fed state. The respiratory exchange ratio $\left(\mathrm{RER}=\mathrm{VCO}_{2} / \mathrm{VO}_{2}\right)$ and heat production $(\mathrm{HP}=$ $\left.(3.185+1.232 \times \mathrm{RER}) \times \mathrm{VO}_{2}\right)$ were calculated .

Histological analysis. For H\&E staining, harvested white or brown adipose tissues and liver were fixed in $4 \%$ (wt/vol) paraformaldehyde and embedded in paraffin. Five-micron-thick tissue sections were deparaffinized, rehydrated, and stained with H\&E. For immunofluorescence staining, harvested epididymal adipose tissues were fixed in $4 \%$ paraformaldehyde in PBS for 1 hour and whole-mounted. After blocking with $5 \%$ goat or donkey serum (Jackson ImmunoResearch) in PBST (0.3\% Triton X-100 in PBS) for 1 hour at room temperature, whole-mounted tissue was incubated overnight with primary antibodies at $4^{\circ} \mathrm{C}$. After several washes with PBST, samples were incubated for 2 hours at room temperature with FITC-conjugated goat anti-rat or TRITC-conjugated goat anti-guinea pig secondary antibody, as appropriate. Samples were stained with 4',6-diamidino-2-phenylindole (DAPI; $1 \mu \mathrm{g} / \mathrm{mL}$ ) to detect nuclei.

Glucose and insulin tolerance tests. For glucose tolerance test (GTT), mice were injected i.p. with $2 \mathrm{~g} / \mathrm{kg}$ D-glucose (MilliporeSigma) after fasting for 16 hours. For insulin tolerance test (ITT), mice were injected i.p. with $1 \mathrm{U} / \mathrm{kg}$ human insulin (Humulin R; Lilly) after fasting for 6 hours. Glucose concentrations were measured in tail vein blood immediately before and 15, 30, 60, and 120 minutes after injection using a Gluco DR TOP glucometer (Allmedicus).

Oral lipid tolerance tests. For lipid tolerance tests, mice were administered corn oil $(10 \mu \mathrm{L} / \mathrm{g})$ by oral gavage after fasting for 16 
hours. Blood samples were collected immediately before and at 1, 2, 3, and 4 hours in tail vein after oral bolus load. Plasma TGs were detected by Triglyceride Reagent (Sigma-Aldrich).

Hyperinsulinemic-euglycemic clamp study. A hyperinsulinemiceuglycemic clamp study was performed in conscious mice 4 days after vein cannulation, as previously described (42). Briefly, after overnight fasting, a 2-hour hyperinsulinemic-euglycemic clamp was performed using a $900 \mathrm{pmol} / \mathrm{kg}$ priming dose and a continuous infusion of human regular insulin (Novolin) at $15 \mathrm{pmol} / \mathrm{min}$, after which $20 \%$ glucose was infused at variable rates to maintain glucose at a constant concentration of 5-6 mM. Insulin-stimulated whole-body glucoseuptake rates were estimated using continuous infusion of [3-3H] glucose $0.1 \mu \mathrm{Ci} / \mathrm{min}$; PerkinElmer Life and Analytical Sciences) throughout clamps. Tissue 2-[$\left[{ }^{14} \mathrm{C}\right] \mathrm{DG}$ 6-phosphate $\left(2-\left[{ }^{14} \mathrm{C}\right] \mathrm{DG}-6-P\right)$ content was determined by separating 2-DG-6-P from 2-DG in supernatants of homogenized tissue samples using an ion exchange column. Insulin-stimulated whole-body glucose-uptake rates were calculated by dividing $\left[{ }^{3} \mathrm{H}\right]$ glucose infusion rates [disintegrations per minute $(\mathrm{dpm} /$ $\mathrm{min})$ ] by the specific activities of plasma glucose $(\mathrm{dpm} / \mu \mathrm{mol})$ during the final 30 minutes of clamps. Hepatic glucose production (HGP) during clamps was determined by subtracting the glucose infusion rate from the whole-body glucose-uptake rate.

In vivo insulin signaling. Overnight-fasted mice were injected i.p. with $1 \mathrm{U} / \mathrm{kg}$ human insulin (Humulin R; Lilly) or an equal volume of saline (control). After 15 minutes, the animals were sacrificed and blood was collected by cardiac puncture. eWAT, liver, and soleus muscle were quickly dissected and frozen in liquid nitrogen for subsequent analysis.

Quantitative RT-PCR. TRIzol reagent (Ambion) was used for total RNA extraction from harvested tissues or primary adipocytes. cDNA was synthesized from $1 \mu \mathrm{g}$ of total RNA using a High Capacity cDNA Reverse Transcription kit (Applied Biosystems). qRT-PCR was performed on a ViiA 7 Real-Time PCR system (Applied Biosystems) using Fast SYBR Green Master Mix (Applied Biosystems) according to the manufacturer's instructions. Expressional profiles were quantified according to the relative ddCt method using 36B4 as a reference gene. The primer sequence is provided in Supplemental Table 2.

Western blot analysis. Whole-cell lysates were prepared by incubating cells in RIPA buffer ( $25 \mathrm{mM}$ Tris- $\mathrm{HCl} \mathrm{pH}$ 7.6, $150 \mathrm{mM} \mathrm{NaCl}$, $1 \%$ NP-40, $1 \%$ sodium deoxycholate, $0.1 \%$ SDS) containing protease and phosphatase inhibitors (Invitrogen). After briefly centrifuging samples, supernatants were collected and assayed for protein concentration using a BCA Protein Assay Kit (Thermo Fisher Scientific). Cell lysates were then mixed with an equal volume of RIPA buffer and boiled for 10 minutes at $95^{\circ} \mathrm{C}$. Samples were then separated by sodium dodecyl sulfate-polyacrylamide gel electrophoresis (SDS-PAGE) and transferred to a polyvinylidene difluoride membrane (Millipore). After blocking in a 5\% BSA solution, the membranes were incubated with the following specific primary antibodies: anti-phospho-HSL (Ser660) (diluted 1:1000; Cell Signaling, catalog 4126), anti-HSL (diluted 1:1000; Cell Signaling, catalog 4107), anti-AKT (diluted 1:1000; Cell Signaling, catalog 9272), anti-phospho-AKT (Ser473) (diluted 1:1000; Cell Signaling, catalog 9271), and anti- $\beta$-actin (diluted 1:1000; Cell Signaling, catalog 3700). The membranes were then washed with Tris-buffered saline containing 0.1\% Tween-20 (TBST) and incubated with horseradish peroxidase-conjugated (HRP-conjugated) anti-rabbit or anti-mouse IgG secondary antibody. Immunoreactive proteins were detected using Western Chemiluminescent HRP
Substrate (ECL) (EMD Millipore), and signals were captured using a ChemiDoc MP system (Bio-Rad).

Glycerol release assay. Lipolysis was quantified as the rate of glycerol release using a free glycerol reagent (MilliporeSigma), following the manufacturer's protocol. Briefly, isolated primary adipocytes in a round-bottom 14-mL tube were incubated with 5-HT or HTR2B agonist (BW 723C86; Tocris) in Krebs Ringer phosphate buffer (136 mM $\mathrm{NaCl}, 4.7 \mathrm{mM} \mathrm{KCl}, 10 \mathrm{mM} \mathrm{NaPO}_{4}, 0.9 \mathrm{mM} \mathrm{MgSO}_{4}, 0.9 \mathrm{mM} \mathrm{CaCl}_{2}$ ) containing $4 \%$ fatty acid-free BSA (MilliporeSigma) for 24 hours. After incubation, $30 \mu \mathrm{L}$ of the cell culture infranatant was mixed with 0.1 $\mathrm{ml}$ of the free glycerol reagent, and the mixture was then incubated at $37^{\circ} \mathrm{C}$ for 5 minutes. The absorbance of the sample at $540 \mathrm{~nm}$ was determined using a spectrophotometer (DU730 Life Science UV/Vis; Beckman Coulter). The amount of glycerol released was expressed relative to the cellular protein content.

Global metabolomics profiling. Global metabolomics profiling of plasma from HFD-fed littermate control and Htr2b-FKO mice was performed by Metabolon, Inc. Plasma samples were processed and frozen at $-70^{\circ} \mathrm{C}$ until analyzed. Compounds were identified by comparison with Metabolon's library of authenticated standards or recurrent unknown entities. The analysis revealed the presence of 827 compounds of known identity (named biochemicals). Enrichment was calculated according to the formula: $(\mathrm{k} / \mathrm{m}) /\{(\mathrm{n}-\mathrm{k}) /(\mathrm{N}-\mathrm{m})\}$, where $\mathrm{k}$ is the number of significantly changed metabolites in a subpathway, $\mathrm{m}$ is the total number of detected metabolites in the subpathway, $\mathrm{n}$ is the total number of significantly changed metabolites, and $\mathrm{N}$ is the total number of detected metabolites. Welch's 2-sample $t$ test was used to identify biochemicals that differed significantly between experimental groups. $P$ less than 0.05 was considered statistically significant.

Quantification of hepatic TGs. Liver tissues were homogenized in 5\% NP-40 using FastPrep-24 (MP Biomedicals). TGs were solubilized by 2 cycles of heating homogenates to $95^{\circ} \mathrm{C}$ for 5 minutes and then cooling to $23^{\circ} \mathrm{C}$. Triglyceride Reagent (Sigma-Aldrich) or PBS was added, and TGs were hydrolyzed into glycerol by incubating samples at $37^{\circ} \mathrm{C}$ for 30 minutes. For colorimetric assay of hydrolyzed TG levels, samples were incubated with Free Glycerol Reagent (Sigma-Aldrich) at $37^{\circ} \mathrm{C}$ for 5 minutes. Differences in absorbance at $540 \mathrm{~nm}$ between hydrolyzed and nonhydrolyzed TGs were quantified using a glycerol standard (Sigma-Aldrich). TG content was normalized to the protein concentration in homogenates, and measured using a BCA Protein Assay Kit (Thermo Fisher Scientific).

Bioinformatic analyses. Bioinformatic analyses using GeneNetwork (http://www.genenetwork.org/) were performed as described in previous studies (43-45). All phenotype data sets in the BXD mouse cohort were downloaded from GeneNetwork. Htr $2 b$ mRNA expression in adipose tissue in the BXD family was searched and analyzed by correlating with published BXD phenotypes. BXD mouse phenotypes showing a significant correlation with $H$ tr $2 b$ expression in murine transcriptome data sets were defined by performing an unbiased bioinformatics analysis. Correlation analyses were based on Pearson product-moment correlation coefficients, determined using the GeneNetwork website. Volcano plots shown are based on Pearson's $r$ and $P$ values. Specific BXD cohort phenotypes showing a significant correlation with Htr $2 b$ expression in adipose tissue (glycemia during GTT in HFD, AST level in HFD, ALT level in HFD) are presented.

Human adipose tissue experiments. We obtained visceral fat tissue from the normal control subjects $(n=10)$ who underwent nephrecto- 
my as a donor in kidney transplantation and visceral fat from the obese patients (non-diabetes mellitus $[\mathrm{DM}]=9, \mathrm{DM}=9$ ) from weight reduction bypass surgery in Seoul National University Bundang Hospital (SNUBH). Baseline characteristics were summarized in Supplemental Table 3. RNA was extracted from frozen human adipose tissue samples using TRIzol (Ambion). For qRT-PCR analysis, $3 \mu \mathrm{g}$ total RNA was reverse transcribed using the High-Capacity cDNA Reverse Transcription kit (Thermo Fisher Scientific). SYBR Green reactions using the SYBR Green PCR Master mix (Enzynomics) were assembled along with $10 \mathrm{pM}$ primers according to the manufacturer's instructions and were performed using the ViiA7 applied biosystems (Thermo Fisher Scientific).

Statistics. All values are expressed as mean \pm SEM, and analyses were performed using SPSS Statistics version 23 (SPSS, Inc.). Two-tailed Student's $t$ test or 1-way ANOVA with post hoc Tukey's test were used to compare groups. $P$ less than 0.05 was considered statistically significant; individual $P$ values are indicated by asterisks in graphs $\left({ }^{*},{ }^{*} P<0.05\right.$,

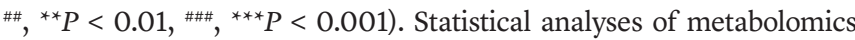
results were performed using Array Studio (Omicsoft) version 7.2 and $\mathrm{R}$ version 3.02 ( $\mathrm{R}$ Foundation for Statistical Computing). Biochemicals in the Metabolon analyses of plasma were rescaled to set the median to 1, based on all samples. Statistical analyses were conducted to detect differences in fold-change (i.e., scaled intensity) in metabolomics data sets.

Study approval. All animal experiments were performed with the approval of the Institutional Animal Care and Use Committee of the KAIST and Yeungnam University. The study in humans was conducted in accordance with the Declaration of Helsinki and was approved by the Ethics Committees of SNUBH (IRB no. B-1801445301 and B-1812513302) and all subjects provided their written informed consent.

\section{Author contributions}

WGC, WC, TJO, SYP, SHC, and HK designed the experiments, wrote the paper, and analyzed data. WGC, IH, SYL, HS, AL, JMS, and $\mathrm{HK}$ conducted the mouse experiments in vivo and in vitro. WGC, WC, and HK performed the metabolomics analysis. WGC, TJO, YKL, SHC, and HK analyzed the human data. WGC, HNC, SYP, and HK performed the CLAMP study. WGC, DR, and HK conducted the bioinformatics analysis of BXD mice. All authors reviewed and approved the manuscript.

\section{Acknowledgments}

We thank Jueun Kim and Hwajin Kim for technical support. We also thank all members of Integrated lab of Metabolism, Obesity, and Diabetes for helpful discussions. This work was supported by grants from the National Research Foundation (NRF) of Korea (NRF-2018R1A2A3074646, NRF-2019R1A5A8083404, and NRF-2020M3A9E4038695 to HK, NRF-2016R1D1A1B04931995 to AL, NRF-2015R1A5A2009124 to SYP).

Address correspondence to: Hail Kim, Graduate School of Medical Science and Engineering, KAIST, 291 Daehak-ro, Yuseong-gu, Daejeon 34141, South Korea. Email: hailkim@kaist.edu. Or to: Sung Hee Choi, Department of Internal Medicine, Seoul National University Bundang Hospital, 173-82 Gumi-ro, Seongnam 13620, South Korea. Email: shchoimd@gmail.com. Or to: So-Young Park, Department of Physiology, College of Medicine, Yeungnam University, 170 Hyunchoongro, Namgu, Daegu 42415, South Korea. Email:sypark@med.yu.ac.kr.
1. Friedrich MJ. Global obesity epidemic worsening. JAMA. 2017;318(7):603.

2. Kahn CR, et al. Altered adipose tissue and adipocyte function in the pathogenesis of metabolic syndrome. JClin Invest. 2019;129(10):3990-4000.

3. Ghaben AL, Scherer PE. Adipogenesis and metabolic health. Nat Rev Mol Cell Biol. 2019;20(4):242-258.

4. Fox CS, et al. Abdominal visceral and subcutaneous adipose tissue compartments: association with metabolic risk factors in the Framingham Heart Study. Circulation. 2007;116(1):39-48.

5 . Walther DJ, et al. Synthesis of serotonin by a second tryptophan hydroxylase isoform. Science. 2003;299(5603):76.

6. Zhang X, et al. Tryptophan hydroxylase-2 controls brain serotonin synthesis. Science. 2004;305(5681):217.

7. Berger M, et al. The expanded biology of serotonin. Annu Rev Med. 2009;60:355-366.

8. Noda M, et al. Multiple signal transduction pathways mediated by 5 -HT receptors. Mol Neurobiol. 2004;29(1):31-39.

9. $\mathrm{Kim} \mathrm{H}$, et al. Serotonin regulates pancreatic beta cell mass during pregnancy. Nat Med. 2010;16(7):804-808.

10. Sumara G, et al. Gut-derived serotonin is a multifunctional determinant to fasting adaptation. Cell Metabolism. 2012;16(5):588-600.

11. Choi W, et al. Serotonin signals through a gutliver axis to regulate hepatic steatosis. Nat Commun. 2018;9(1):4824.
12. Crane JD, et al. Inhibiting peripheral serotonin synthesis reduces obesity and metabolic dysfunction by promoting brown adipose tissue thermogenesis. Nat Med. 2015;21(2):166-172.

13. Oh CM, et al. Regulation of systemic energy homeostasis by serotonin in adipose tissues. Nat Commun. 2015;6:6794.

14. Paulmann N, et al. Intracellular serotonin modulates insulin secretion from pancreatic beta-cells by protein serotonylation. PLoS Biol. 2009;7(10):e1000229.

15. Coelho WS, et al. Serotonin stimulates mouse skeletal muscle 6-phosphofructo-1-kinase through tyrosine-phosphorylation of the enzyme altering its intracellular localization. Mol Genet Metab. 2007;92(4):364-370.

16. Hajduch E, et al. Serotonin (5-Hydroxytryptamine), a novel regulator of glucose transport in rat skeletal muscle. J Biol Chem. 1999;274(19):13563-13568.

17. Shong KE, et al. Serotonin regulates de novo lipogenesis in adipose tissues through serotonin receptor 2A. Endocrinol Metab. 2020;35(2):470-479.

18. Hwang I, et al. GABA-stimulated adiposederived stem cells suppress subcutaneous adipose inflammation in obesity. Proc Natl Acad Sci U S A. 2019;116(24):11936-11945.

19. Li H, et al. An integrated systems genetics and omics toolkit to probe gene function. Cell Syst. 2018;6(1):90-102.

20. Zechner R, et al. Cytosolic lipolysis and lipophagy: two sides of the same coin. Nat Rev Mol Cell
Biol. 2017;18(11):671-684

21. Milbourne EA, Bygrave FL. Do nitric oxide and cGMP play a role in calcium cycling? Cell Calcium. 1995;18(3):207-213.

22. Katona D, et al. Calcium-dependent mitochondrial cAMP production enhances aldosterone secretion. Mol Cell Endocrinol. 2015;412:196-204.

23. Cameron EG, Kapiloff MS. Intracellular compartmentation of cAMP promotes neuroprotection and regeneration of CNS neurons. Neural Regen Res. 2017;12(2):201-202.

24. Lafontan M, Langin D. Lipolysis and lipid mobilization in human adipose tissue. Prog Lipid Res. 2009;48(5):275-297.

25. Sengenes $\mathrm{C}$, et al. Involvement of a cGMP dependent pathway in the natriuretic peptide-mediated hormone-sensitive lipase phosphorylation in human adipocytes. J Biol Chem. 2003;278(49):48617-48626.

26. Roden M, Shulman GI. The integrative biology of type 2 diabetes. Nature. 2019;576(7785):51-60.

27. Donnelly KL, et al. Sources of fatty acids stored in liver and secreted via lipoproteins in patients with nonalcoholic fatty liver disease. J Clin Invest. 2005;115(5):1343-1351.

28. Namkung J, et al. Inhibition of serotonin synthesis induces negative hepatic lipid balance. Diabetes Metab J. 2018;42(3):233-243.

29. Young RL, et al. Augmented capacity for peripheral serotonin release in human obesity. Int JObes (Lond). 2018;42(11):1880-1889.

30. Fukui M, et al. High plasma 5-hydroxyindole-3- 
acetic acid concentrations in subjects with metabolic syndrome. Diabetes Care. 2012;35(1):163-167.

31. Girousse A, et al. Partial inhibition of adipose tissue lipolysis improves glucose metabolism and insulin sensitivity without alteration of fat mass. PLoS Biol. 2013;11(2):e1001485.

32. Schoiswohl G, et al. Impact of reduced ATGLmediated adipocyte lipolysis on obesity-associated insulin resistance and inflammation in male mice. Endocrinology. 2015;156(10):3610-3624.

33. Friedman SL, et al. Mechanisms of NAFLD development and therapeutic strategies. Nat Med. 2018;24(7):908-922.

34. Ebrahimkhani MR, et al. Stimulating healthy tissue regeneration by targeting the $5-\mathrm{HT}(2)$ $\mathrm{B}$ receptor in chronic liver disease. Nat Med. 2011;17(12):1668-1673.

35. Fournier JC, et al. Antidepressant drug effects and depression severity: a patient-level meta-analysis. JAMA. 2010;303(1):47-53.

36. Lyseng-Williamson KA. Telotristat ethyl: a review in carcinoid syndrome diarrhoea. Drugs. 2018;78(9):941-950.

37. Miyazaki M, et al. Sarpogrelate hydrochloride, a selective 5-HT2A antagonist, improves vascular function in patients with peripheral arterial disease. J Cardiovasc Pharmacol. 2007;49(4):221-227.

38. Bohula EA, et al. Cardiovascular safety of lorcaserin in overweight or obese patients. $N$ Engl J Med. 2018;379(12):1107-1117.

39. Tramer MR, et al. A quantitative systematic review of ondansetron in treatment of established postoperative nausea and vomiting. $B M J$. 1997;314(7087):1088-1092.

40. Omer A, Quigley EMM. An update on prucalopride in the treatment of chronic constipation.
Therap Adv Gastroenterol. 2017;10(11):877-887.

41. Eguchi J, et al. Transcriptional control of adipose lipid handling by IRF4. Cell Metab. 2011;13(3):249-259.

42. Cha HN, et al. Peroxiredoxin 2 deficiency aggravates aging-induced insulin resistance and declines muscle strength. J Gerontol A Biol Sci Med Sci. 2019;74(2):147-154.

43. Andreux PA, et al. Systems genetics of metabolism: the use of the BXD murine reference panel for multiscalar integration of traits. Cell. 2012;150(6):1287-1299.

44. Ryu D, et al. A SIRT7-dependent acetylation switch of GABPbeta1 controls mitochondrial function. Cell Metab. 2014;20(5):856-869.

45. Li H, Auwerx J. Mouse systems genetics as a prelude to precision medicine. Trends Genet. 2020;36(4):259-272. 